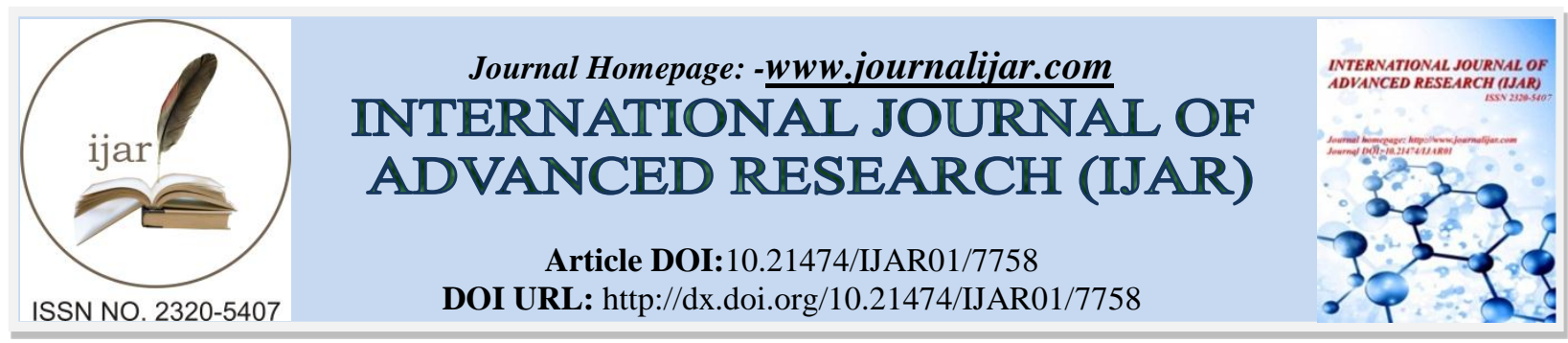

RESEARCH ARTICLE

\title{
REVERSIBLE LEFT VENTRICULAR NONCOMPACTION AFTER PREGNANCY: A PHYSIOLOGICAL CARDIOMYOPATHY.
}

Majdoub Kawtar, Guennoun Zineb, Lagziri Alae Eddine, Doghmi Nawal and Cherti Mohamed. Department of cardiology B, CHU Ibn Sina, Mohammed V University, Rabat, Morocco.

\section{Manuscript Info}

Manuscript History

Received: 24 July 2018

Final Accepted: 30 August 2018

Published: September 2018

Keywords:-

Left ventricular noncompaction,

reversibility, pregnancy.

\begin{abstract}
Patients with chronic anemia, athletes and pregnant women may develop left ventricular trabeculations that may be consistent with the diagnosis of left ventricular noncompaction (LVNC). We report the case of a pregnant woman who was found to have LV trabeculations consistent with LVNC, which regressed in postpartum follow-up. This observation is interesting to distinguish between LVNC as a disease, and non-compaction syndrome that could be associated with some cardiomyopathies or some physiological states such as pregnancy.
\end{abstract}

Copy Right, IJAR, 2018. All rights reserved.

\section{Introduction:-}

Left ventricular noncompaction (LVNC) is a rare cardiomyopathy that is thought to be caused by abnormal myocardial embryogenesis in utero. Besides, there is a distinct "left ventricular noncompaction" phenotype found in some patients with congenital heart and neuromuscular diseases, or in some particular situations, as pregnancy or intensive physical training, where de novo trabeculations would occur consequently to an increase in cardiac output and in stroke volume. In post-partum, these trabeculations are expected to regress after normalization of blood volume $(1,2)$, as illustrated in the case we report.

\section{Observation:-}

A 28-year-old woman was referred to cardiology consultation for atypical chest pain and palpitations. The patient had history of systemic lupus erythematosus with neurological manifestations, and a normal pregnancy one year before. Her physical examination was normal except for faint heart sounds. Her electrocardiogram showed low QRS voltage. The transthoracic echocardiogram (TEE) revealed the presence of a circumferential pericardial effusion of moderate abundance, more abundant next to the left ventricle, with no evidence of haemodynamic compromise. The parasternal short-axis view showed a hyper-trabeculated left ventricle with a 'spongy' look (Figure 1, A), and an end systolic ratio of noncompacted to compacted layers above 2 as described by Jenni et al. (2) (Figure 1, B). Color Doppler evidenced deeply perfused intertrabecular recesses. Left ventricular size and function were normal with global longitudinal strain (GLS) at -22\% (Figure 1, C).

The cardiac magnetic resonance imaging (CMR) confirmed the LVNC diagnosis with an end diastolic short-axis noncompacted to compacted myocardial ratio greater than 3 as described by Stacey et al. (3) and Grothoff et al. (4) (Figure 1, D).

During follow-up, we repeated the TTE and the CMR a few months later, we noticed a clear regression of the trabeculations, with normalization of the noncompacted to compacted myocardial ratio (Figure $2 \mathrm{~A}, \mathrm{~B}$ ). 


\section{Discussion:-}

LVNC was first reported as an isolated condition in 1984 by Engberding et al. who described this abnormal observation as the persistence of myocardial sinusoids (6). This rare cardiomyopathy was later, in 1995, included in the group of « unclassified» cardiomyopathies by the World Health Organization (7), which was approved by the European Society of Cardiology Working Group on Myocardial and Pericardial Disease that stated in 2008 that "it is not clear whether LVNC is a separate cardiomyopathy, or merely a congenital or acquired morphologic trait shared by many phenotypically distinct cardiomyopathies" (8). In contrast, in 2006, the American Heart Association had classified LVNC as primary, genetic cardiomyopathy (9).

However, it is necessary to distinguish the LVNC disorder, non-reversible, and the "LVNC" syndrome where hypertrabeculations are reversible. The LVNC disorder would be the consequence of an alteration of the myocardial structure secondary to incomplete embryogenesis (10). Otherwise, the "LVNC" phenotype reported in pregnant women or in athletes as de novo trabeculations would occur as the result of an increase in cardiac output and in stroke volume.

This case illustrates one of those acquired "LVNC" syndrome, observed during pregnancy, which regressed after normalization of stroke volume.

Sabiha et al. (1) evaluated 102 primigravida pregnant women with a series of echocardiograms in the first and third trimesters and in postpartum, and found de novo appearance of LV trabeculations in $25 \%$ of pregnant women, which completely regressed in $69 \%$ of them over a follow-up period of 1 year. This phenomenon had also been reported in patients followed for chronic anemia (11) and in highly trained athletes (12) who may develop LV trabeculations fulfilling echocardiographic LVNC criteria.

The common factor in the three situations is the increase in preload. These different studies suggest therefore that the onset of LV trabeculations occurs in response to an increase in preload and are not specific to LVNC disorder. Thereby, these factors must be taken into consideration in the presence of LV trabeculations outside the context of heart failure or familial cardiomyopathy.

The clinical presentation of LVNC disorder is highly variable, ranging from asymptomatic patients to heart failure, arrhythmia or thromboembolic complications. Hence, the diagnosis is often delayed due to limited knowledge of clinical features and imaging criteria of LVNC (13).

The diagnosis of LVNC is based on echocardiography and is characterized by the presence of numerous trabeculations separated by deep recesses, in hypertrophied and often hypokinetic LV segments. The most frequent localization of LVNC are the apex, the lateral and inferior walls (14). Despite the absence of a gold standard for LVNC diagnosis, cardiac magnetic resonance imaging is aknowledged by many cardiologists to be the best tool for investigating the presence of LVNC criteria (15).

The prognosis of LVNC is not well established. Patients may remain asymptomatic for a long time without embolic complications nor ventricular arrhythmias.

The therapeutic management is not codified. It is primarily symptomatic treating signs of heart failure. The preventive approach is very discussed. Anticoagulants are necessary in case of high risk of thromboembolic events. The systematic implantation of a defibrillator to prevent arrhythmias should be evaluated. Cardiac transplantation, especially in young people, is not a commonly accepted recommendation. However, in case of failure of the medical treatment, the implantation of a pacemaker or even a heart transplantation should be considered (16). Finally, firstdegree family members of all patients diagnosed with LVNC should have a screening echocardiogram.

\section{Conclusion:-}

The LVNC is an entity that still seeks its place in cardiomyopathies and whose diagnosis may be under or overdetected. Hence, it is very important to distinguish the LVNC disorder of non-reversible genetic origin and the noncompaction syndrome which is an acquired reversible hypertrabeculations. 


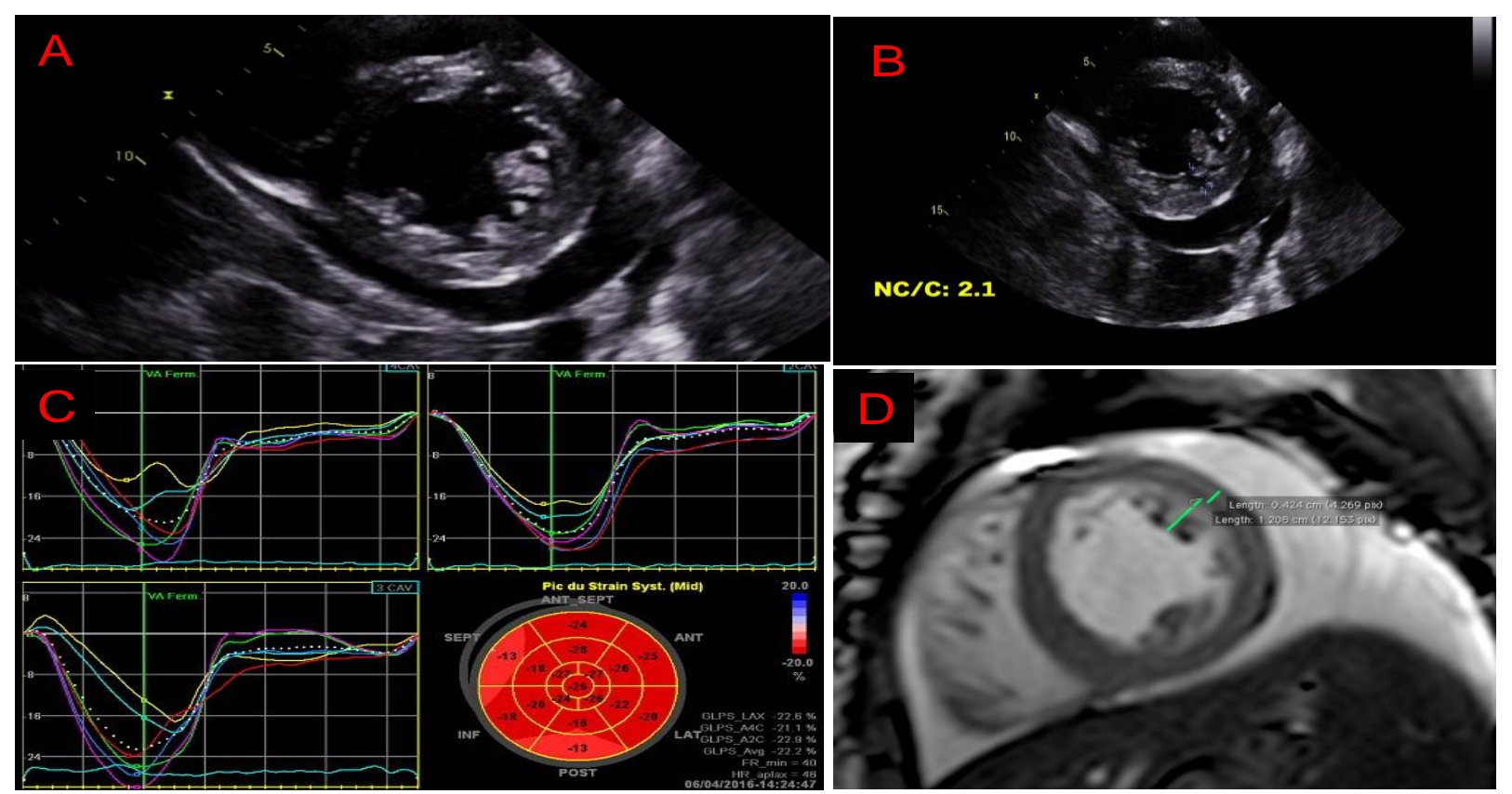

Figure 1:- A: Parasternal long axis view on TTE showing LV trabeculations; B: End systolic ratio of noncompacted to compacted layers at 2.1 on TTE; C: Global longitudinal strain at $-22 \%$, D: Short axis view on CMR, End systolic ratio of noncompacted to compacted layers at 3
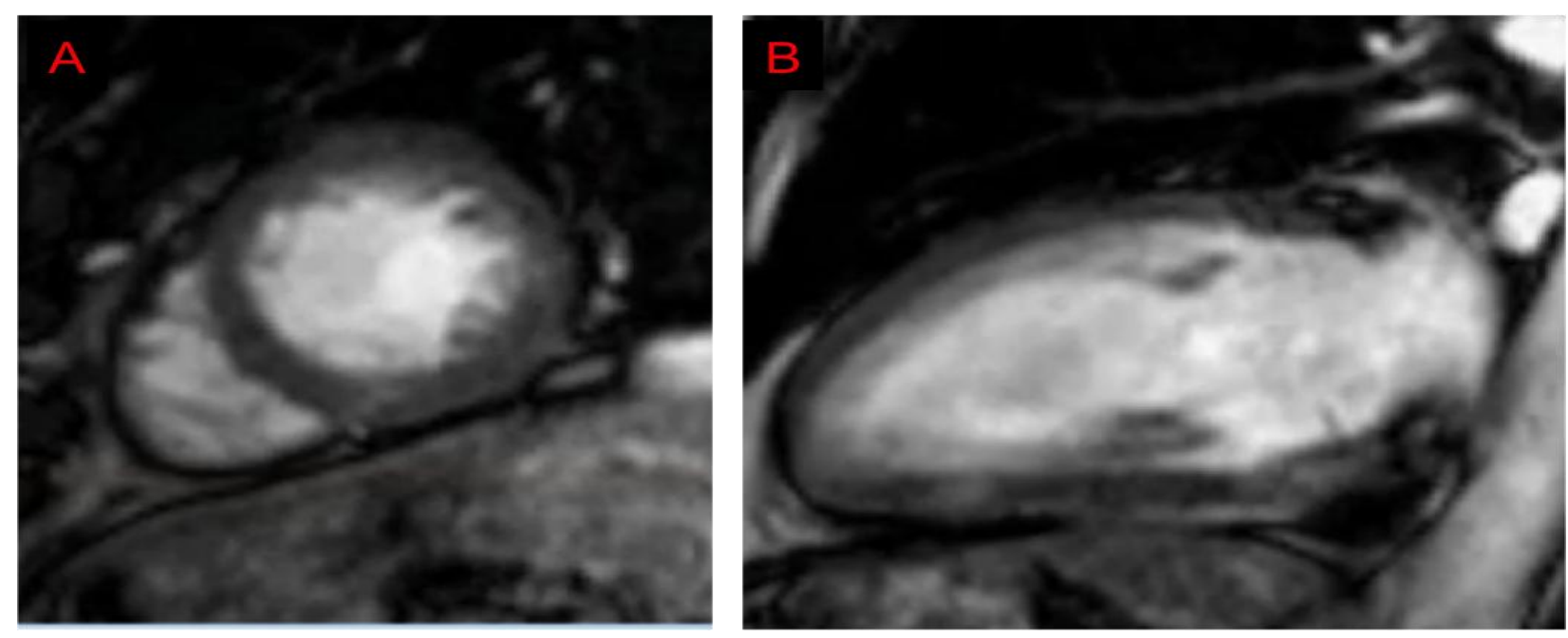

Figure 2:- A and B: Short axis and long axis views on CMR showing the regression of LV trabeculations 


\section{References:-}

1. Gati, Sabiha, et al. "Reversible de novo left ventricular trabeculations in pregnant women: implications for the diagnosis of left ventricular non-compaction in low risk populations". Circulation (2014):114.

2. Asfalou, Iliyasse, et al. "Left ventricular noncompaction-A rare form of cardiomyopathy: Revelation modes and predictors of mortality in adults through 23 cases". Journal of the Saudi Heart Association 29.2 (2017): 102109.

3. Jenni, R., et al. "Echocardiographic and pathoanatomical characteristics of isolated left ventricular noncompaction: a step towards classification as a distinct cardiomyopathy". Heart 86.6 (2001): 666-671.

4. Stacey, R. Brandon, et al. "Comparison of systolic and diastolic criteria for isolated LV noncompaction in CMR". JACC: Cardiovascular Imaging 6.9 (2013): 931-940.

5. Grothoff, Matthias, et al. "Value of cardiovascular MR in diagnosing left ventricular non-compaction cardiomyopathy and in discriminating between other cardiomyopathies". European radiology 22.12 (2012): 2699-2709.

6. Engberding, Rolf. "Identification of a rare congenital anomaly of the myocardium by two-dimensional echocardiography: Persistence of isolated myocardial sinusoinds". Am J Cardiol53 (1984): 1733-1734.

7. Richadson, P. "Report of the 1995 World Health Organization/International Society and Federation of Cardiology. Task force on the definition and classification of cardiomyopathies". Circulation 93 (1996): 841842 .

8. Elliott, Perry, et al. "Classification of the cardiomyopathies: a position statement from the European Society Of Cardiology Working Group on Myocardial and Pericardial Diseases". European heart journal 29.2 (2007): 270276.

9. Maron, Barry J., et al. "Contemporary definitions and classification of the cardiomyopathies: an American heart association scientific statement from the council on clinical cardiology, heart failure and transplantation committee; quality of care and outcomes research and functional genomics and translational biology interdisciplinary working groups; and council on epidemiology and prevention". Circulation 113.14 (2006): 1807-1816.

10. Thavendiranathan P, Chow Cg.La non- compaction ventriculaire isolée : une nouvelle cardiomyopathie. Compte rendu des conférences scientifiques de la division de cardiologie, hôpital St. Michael, Université de Toronto, 2008 ; volume XIII, numéro 3.

11. Gati, S., et al. "Increased left ventricular trabeculation in individuals with sickle cell anaemia: physiology or pathology?". International journal of cardiology 168.2 (2013): 1658-1660.

12. Gati, Sabiha, et al. "Increased left ventricular trabeculation in highly trained athletes: do we need more stringent criteria for the diagnosis of left ventricular non-compaction in athletes?". Heart 99.6 (2013): 401-408.

13. Gorincour, G., et al. "Cardiomyopathies par non compaction du ventricule gauche". Journal de Radiologie 85.12 (2004): 2033-2034.

14. Ikeda, Uichi, Masatoshi Minamisawa, and Jun Koyama. "Isolated left ventricular non-compaction cardiomyopathy in adults". Journal of cardiology 65.2 (2015): 91-97.

15. Petersen, Steffen E., et al. "Left ventricular non-compaction: insights from cardiovascular magnetic resonance imaging". Journal of the American College of Cardiology 46.1 (2005): 101-105.

16. Kohli, Sanjay K., et al. "Diagnosis of left-ventricular non-compaction in patients with left-ventricular systolic dysfunction: time for a reappraisal of diagnostic criteria?". European heart journal 29.1 (2007): 89-95. 